\title{
Clinical Application of Liquid Chromatography Tandem Mass Spectrometry Using Dried Blood Spot as a More Rapid Method for Determination of Methylmalonic Acid, Propionylcarnitine, and Total Homocysteine \\ Journal of Inborn Errors \\ of Metabolism \& Screening 2020, Volume 8: e20190005 \\ DOI: 10.1590/2326-4594-JIEMS-2019-0005
}

\author{
Hiroyuki lijima $^{1}\left(\mathbb{D}\right.$, Nobuyuki Ishige $^{2}$ and Mitsuru Kubota ${ }^{1}$
}

\begin{abstract}
Methylmalonic acidemia (MMA) should be diagnosed in early infancy and receive appropriate management promptly after the diagnosis to prevent severe complications leading to death. At present, a newborn screening (NBS) method using tandem mass spectrometry (MS/MS) identifies suspected patients with MMA by elevated propionylcarnitine. In addition, a liquid chromatography tandem mass spectrometry (LC/MS/MS) method using dried blood spot is effective to detect some metabolites as a second-tier test, and reduces the false-positive rate in NBS. However, these tests were only used in screening, and not applied as an examination for evaluating treatment. Herein, we describe a 57-day-old girl with MMA under treatment with cobalamin who had elevated urinary methylmalonic acid levels. We applied the LC/MS/MS method with a separation column to evaluate her cobalamin responsiveness, and discovered an insufficient cobalamin dose earlier than would have been possible using other methods. Based on the current data, this method seems to be applicable for the follow-up of the treatment of MMA patients. However, this should be confirmed with more experience with a larger number of cases and a wider spectrum of disorders.
\end{abstract}

\section{Keywords}

cobalamin responsiveness, liquid chromatography tandem mass spectrometry, methylmalonic acidemia, newborn screening.

\section{Introduction}

Methylmalonic acidemia (MMA) is a relatively common autosomal recessive inherited disorder caused by a deficiency of methylmalonyl-CoA mutase (MCM) or its cofactor adenosylcobalamin. Methylmalonyl-CoA apoenzyme deficiencies are subdivided into two subgroups, i.e., mut defect with some residual activity in the presence of high concentrations of adenosylcobalamin and $m u t^{0}$ defect with undetectable activity.[1] Patients with MMA display elevation of levels of propionylcarnitine (C3), methylmalonic acid, methylcitric acid, and 3-hydroxypropionic acid. These metabolites are derived from the propionate pathway, namely, isoleucine, valine, threonine, methionine, odd-chain fatty acids, and cholesterol metabolism. [2] Although the clinical presentation of patients with MMA is variable, the principal symptoms are vomiting, metabolic acidosis, hyperammonemia, and encephalopathy that could cause death. In addition, MMA patients with neonatal onset usually have a severe clinical course.[1,3-4] Even under appropriate metabolic management, patients with MMA can experience metabolic attacks and multisystemic disorders, including progressive renal disease,[3-4] cardiomyopathy,[5]

\footnotetext{
${ }^{1}$ National Center for Child Health and Development, Department of Genera Pediatrics \& Interdisciplinary Medicine, 2-10-1 Okura, Setagaya-ku, Tokyo 157-8535, Japan

2 Tokyo Health Service Association, Division of Newborn Screening, 1-11-1 Ichigayacho, Shinjuku-ku, Tokyo 162-0843, Japan
}

Received May 09, 2019, and in revised form October 30, 2019. Accepted for publication December 16, 2019.

\section{Corresponding Author:}

Mitsuru Kubota, Department of General Pediatrics \& Interdisciplinary Medicine, National Center for Child Health and Development, 2-10-1 Okura, Setagayaku, Tokyo 157-8535, Japan.

Email: kubota-mt@ncchd.go.jp 
neurodevelopmental disorders, [6-7] and pancytopenia.[8] These complications increase the morbidity and mortality rate in infancy and childhood. $[1,4]$ To prevent this tragic progression, MMA should be diagnosed in early infancy and continue to receive appropriate management.

Recently, newborn screening (NBS) using tandem mass spectrometry (MS/MS) identifies suspected patients with MMA by elevated $C 3,[9-10]$ and effective new second-tier tests shorten the diagnostic process.[11-13] Although these tests improve the accuracy and speed of screening, they are not usually applied as an examination for evaluating treatment.

Herein, we describe a simple liquid chromatography tandem mass spectrometry (LC/MS/MS) method using dried blood spot (DBS) with a separation column, which can provide the acylcarnitine profile, methylmalonic acid level, and total homocysteine (tHcy) level in the same sample. The present article aimed to evaluate the effectiveness of this method for diagnosis and treatment of MMA.

\section{Case Report}

A 57-day-old Japanese girl with MMA was referred to our hospital because of the family's recent relocation to Japan. She was the first child born to non-consanguineous Japanese parents, and had no significant family history. She was born at 38 weeks after an uneventful pregnancy in Dubai, where NBS identified elevated C3 $(6.70 \mu \mathrm{mol} / \mathrm{L}$; reference interval, $<3.60 \mu \mathrm{mol} / \mathrm{L})$. At 12 days old, she had elevated C3 $(9.17 \mu \mathrm{mol} / \mathrm{L})$ and increased ratio with acetylcarnitine $(\mathrm{C} 3 / \mathrm{C} 2)(0.57$; reference interval, $<0.25)$ on a secondary screening test. At 30 days old she was hospitalized to follow up the abnormal NBS results. A urinary organic acid analysis revealed a massive level of methylmalonic acid (8698 $\mu \mathrm{mol} / \mathrm{mmol}$ creatinine; reference interval, $<11 \mu \mathrm{mol} / \mathrm{mmol}$ creatinine), without metabolic acidosis. Her ammonia level (62 $\mu \mathrm{mol} / \mathrm{L})$, cobalamin level $(996 \mathrm{pg} / \mathrm{mL})$, and serum homocysteine level $(6.3 \mu \mathrm{mol} / \mathrm{L})$ were normal. The acylcarnitine profile showed low free carnitine $(11.7 \mu \mathrm{mol} / \mathrm{L}$; reference interval, $27-49 \mu \mathrm{mol} / \mathrm{L})$, and high acylcarnitine level $(32.1 \mu \mathrm{mol} / \mathrm{L}$; reference interval, 7-19 $\mu \mathrm{mol} / \mathrm{L}$ ). MMA was diagnosed and the patient was started on hydroxycobalamin injection $1 \mathrm{mg} /$ day and oral L-carnitine $100 \mathrm{mg} / \mathrm{kg} / \mathrm{day}$. Urinary organic acid analyses, repeated at 4 and 10 days after initiation of treatment, showed decreased methylmalonic acid (4735 and $2823 \mu \mathrm{mol} / \mathrm{mmol}$ creatinine, respectively).

When the patient returned to Japan and was admitted to our hospital, her height and weight were $54.4 \mathrm{~cm} \mathrm{(-} 1.0$ $\mathrm{SD})$ and $4.59 \mathrm{~kg}(-1.0 \mathrm{SD})$, respectively. She was developing normally with protein-restricted milk $(92 \mathrm{kcal} / 100 \mathrm{~mL}$, natural protein $0.5 \mathrm{~g} / 100 \mathrm{~mL}$ ). She did not present with any symptoms (hepatosplenomegaly, muscle weakness, or neurological abnormalities). Methylmalonic acid in urine was still high $(0.432 / 10 \mu \mathrm{g}$ HDA; reference interval, < 0.007/10 $\mu \mathrm{g}$ HDA; heptadecanoic acid [HDA] as an internal standard) in spite of drug treatment and dietary restriction. Laboratory data indicated normal levels of serum transaminases (AST 26 IU/L and ALT $20 \mathrm{IU} / \mathrm{L})$ and ammonia $(57 \mu \mathrm{mol} / \mathrm{L})$, with no metabolic derangements such as hypoglycemia, hyperlipidemia, hyperuricemia, or hyperlactacidemia. Because of the high value of urinary methylmalonic acid with the previous dose of cobalamin, we increased the dose of cobalamin from $1 \mathrm{mg} /$ day to $10 \mathrm{mg} /$ day to assess cobalamin responsiveness accurately.

\section{Materials and Methods}

Written informed consent was obtained from the patient's parents for all procedures and tests described herein, per the tenets of the Declaration of Helsinki, in addition to consent for publication of the patient's data.

\section{MS/MS analysis}

The primary NBS assay that measures amino acids and acylcarnitines was carried out using the flow injection method (no separation column in the system; FI/MS/MS). The analysis was performed by a TQ Detector coupled with a $1525 \mu$ binary HPLC pump and 2777C sample manager (Waters, Milford, MA, USA). LC/MS/MS analysis was performed by a Nexera HPLC system, coupled with an LCMS-8050 (Shimadzu Corporation, Kyoto, Japan) for the second-tier test, tHcy and methylmalonic acid analysis on DBS. Acylcarnitines and LC/MS/MS (assays of tHcy and methylmalonic acid) were analyzed separately, in three different assays from three separately punched DBSs from a single sample.

Electrospray ionization source was operated in both negative and positive mode for methylmalonic acid and the others, respectively. The LC separations were carried out on a SUPELCO Discovery HS F5-3 $(150 \times 2.1 \mathrm{~mm}, 3 \mu \mathrm{m})$ from Sigma-Aldrich Japan K.K. (Tokyo, Japan) and a Scherzo SS-C18 ion exchange multi-mode ODS column $(150 \times 3 \mathrm{~mm}, 3 \mu \mathrm{m})$ from Imtakt Corporation (Kyoto, Japan) for tHcy and methylmalonic acid, respectively. Sample preparation for the second-tier test was carried out using modification of the method by Turgeon et al.[14] LC conditions of tHcy and methylmalonic acid are shown in Supplementary Tables S1 and S2, respectively. The instrument was optimized automatically by an internal algorithm to monitor the transitions m/z 136.00 to m/z 90.1 and m/z 140.0 to m/z 94.10 for unlabeled and deuterium-labeled (d4) Hcy, and m/z 117.10 to $\mathrm{m} / \mathrm{z} 73.15$ and $\mathrm{m} / \mathrm{z} 120.10$ to $\mathrm{m} / \mathrm{z} 76.20$ for unlabeled and deuterium-labeled (d3) methylmalonic acid. D4-Hcy was converted from $\mathrm{d} 8$-homocystine after reduction by dithiotreitol added to the reaction solution. The results were acquired using LabSolutions Version 5.91 and LabSolutions Insight LCMS Version 3.10 (Shimadzu Corporation, Kyoto, Japan). Injection volume was $10 \mu \mathrm{L}$ and $2 \mu \mathrm{L}$ for acylcarnitines and LC/MS/MS, respectively. 


\section{Analysis of MCM activity}

MCM activity was analyzed using a previously described method based on ultrahigh-performance LC/MS/MS.[15] MCM was obtained from $5 \times 10^{5}$ lymphocytes in Tris-sulfate buffer $(\mathrm{pH}$ 7.5) lysed by sonic disruption, and then adenosylcobalamin was added to the resultant lysate. After this solution was warmed at $37^{\circ} \mathrm{C}$ for a few minutes, it was spiked with methylmalonyl-CoA and incubated at $37^{\circ} \mathrm{C}$ for $15 \mathrm{~min}$. The reaction was quenched with $\mathrm{HClO}_{4}$, and acetyl-CoA (Internal Standard solution) was added. The solution was centrifuged, and the supernatant was analyzed by ultrahigh-performance LC/MS/MS to determine produced succinyl-CoA. MCM activity was evaluated using quantitation of succinyl-CoA produced.

\section{Urinary organic acid analysis}

The urine samples were spiked with $10 \mu \mathrm{g}$ heptadecanoic acid (HDA) and 3-hydroxy myristic acid as internal standards, and treated with urease to remove interfering urea by a procedure extensively modified from that of Matsumoto and Kuhara.[16] The volume of urine including $20 \mu \mathrm{g}$ of creatinine was used. Protein was precipitated with $900 \mu \mathrm{L}$ of ethanol and removed by centrifugation. The deproteinized solution was then evaporated to dryness. Compounds of interest in the dried residue were converted to trimethylsilyl derivatives with $100 \mu \mathrm{L}$ of N,O-Bis (trimethylsilyl) trifluoroacetamide with chlorotrimethylsilane and analyzed by gas chromatography/mass spectrometry.

\section{Mutation analysis}

Genomic DNA was extracted from the peripheral blood leukocytes of the patient. A mutation analysis of $M M U T$, PCCA, PCCB, ABCD4, HCFC1, LMBRD1, MMAA, MMAB, $M M A C H C$, and $M M A D H C$ was performed by next-generation sequencing (Nextseq Sequencing System, Illumina, San Diego, CA, USA) at the Kazusa DNA Research Institute. Common genetic variations were identified using the following public databases: Exome Aggregation Consortium (ExAC), NCBI ClinVar, Human Genetic Variation Database (HGVD), and Human Gene Mutation Database (HGMD).

\section{In silico analysis}

Phylogenic information was obtained via Vertebrate Multiz Alignment \& Conservation in the UCSC Genome Browser (https://genome.ucsc.edu/). We used PolyPhen-2 (http://genetics. bwh.harvard.edu/pph2/) and PROVEAN and SIFT (http:// provean.jcvi.org/index.php) predictive algorithms to evaluate the pathogenicity of the identified sequence variants.

\section{Results}

The results indicated that $\mathrm{C} 3, \mathrm{C} 3 / \mathrm{C} 2$, and methylmalonic acid levels were decreased on DBS, although tHcy was stable at the normal value. A urinary organic acid analysis also revealed a decrease in methylmalonic acid. Plasma amino acid analysis could not detect homocysteine. These examinations were performed simultaneously 14 days after increasing the dose of cobalamin (Table 1). The LC/MS/MS method took 3-5 days until results were given to physicians, whereas the urinary organic acid analysis took 2-3 weeks.

The amounts of produced succinyl-CoA in the patient were low (mean $0.35 \mathrm{pmol} / \mathrm{min} / 5.0 \times 10^{5}$; 0.332 and 0.372 $\left.\mathrm{pmol} / \mathrm{min} / 5.0 \times 10^{5}\right)$ compared with those in control samples (mean $42.67 \mathrm{pmol} / \mathrm{min} / 5.0 \times 10^{5} ; 40.97,42.08$, and $44.97 \mathrm{pmol} /$ $\min / 5.0 \times 10^{5}$ ). These data revealed that MCM activity in our patient was $0.82 \%$ of the control.

Next-generation sequencing revealed heterozygous missense mutations (c.1292T $>$ C [p.Met431Thr, located in exon 6] and c.1808G $>$ A [p.Arg603Lys, located in exon 10]) in a responsible gene, MMUT, of the patient. The former mutation was novel, whereas the latter was previously reported as $m u t^{0}$ MMA.

Table 1. Laboratory data for the case before and after cobalamin increase

\begin{tabular}{|c|c|c|c|c|c|c|c|c|}
\hline Age (months) & & 1 & 2 & 3 & 7 & 13 & 18 & \\
\hline Natural protein (g/kg/day) & & - & 0.8 & 0.8 & 0.9 & 1.6 & 1.8 & $\begin{array}{l}\text { Cut-off } \\
\text { value }\end{array}$ \\
\hline Cobalamin (mg/day) & & 0 & 1 & 10 & 10.5 & 10.5 & 10.5 & \\
\hline \multirow{2}{*}{$\mathrm{MS} / \mathrm{MS}$ in DBS $(\mu \mathrm{mol} / \mathrm{L})$} & $\mathrm{C} 3 / \mathrm{C} 2$ & 0.57 & 0.37 & 0.08 & 0.20 & 0.28 & 0.28 & $<0.25$ \\
\hline & MMA & - & 11.46 & 1.02 & - & - & - & $<3.00$ \\
\hline Urinary organic acid analysis (/10 $\mu \mathrm{g}$ HDA) & MMA & - & 0.432 & 0.043 & 0.295 & 0.434 & 0.373 & $<0.007$ \\
\hline Plasma amino acid analysis (nmol/mL) & tHcy & 6.3 & - & ND & - & - & - & $>15.0$ \\
\hline
\end{tabular}

FI/MS/MS, flow injection tandem mass spectrometry; LC/MS/MS, liquid chromatography tandem mass spectrometry; DBS, dried blood spot; MMA, methylmalonic acid; tHcy, total homocysteine; ND, not detected 
[17-19] According to ClinVar, ExAC, HGVD, and HGMD, p.Met431Thr was not listed. Both Met431 and Arg603 are conserved throughout species. Upon PolyPhen-2, PROVEAN, and SIFT analyses, p.Met431Thr was predicted as "probably damaging", "deleterious", and "damaging". However, PolyPhen-2, PROVEAN and SIFT analyses predicted p.Arg603Lys to be "benign", "neutral", and "tolerated" respectively.

At present, the patient is 18 months old and developing normally with no symptoms, metabolic acidosis, or hyperammonemia under high-dose cobalamin $(10.5 \mathrm{mg} /$ day $)$, oral L-carnitine $(50 \mathrm{mg} / \mathrm{kg} /$ day $)$, and mild dietary restriction (natural protein $1.8 \mathrm{~g} / \mathrm{kg} /$ day).

\section{Discussion}

The patient's disease was diagnosed as MMA and she was started on treatment with a combination of cobalamin and carnitine, but the value of her urinary methylmalonic acid remained high because of the insufficient dose of cobalamin in Dubai. Using the LC/MS/MS method and DBS with the separation column, we could quickly determine cobalamin responsiveness in this case and relieve dietary restrictions by increasing the dose of cobalamin. Althogh the values of $\mathrm{C} 3$ and urinary methylmalonic acid increased after alleviation of protein restriction, these data at 18 months (natural protein $1.8 \mathrm{~g} / \mathrm{kg} /$ day) were lower than before increasing the dose of cobalamin (natural protein 0.8 $\mathrm{g} / \mathrm{kg} /$ day). In addition, our patient's growth and development are good, and she has had no clinical symptoms. Therefore, we believe that reintensification of protein restriction is not necessary under high-dose cobalamin.

There is a few report on the evaluation of high doses cobalamin for MMA patients. Recent case series (3 cbl C patients, $1 \mathrm{cbl}$ A patient, and $1 \mathrm{cbl}$ IX patient ) showed that higher cobalamin dose produced biochemical responses such as lower range plasma tHcy and higher methionine values as well as low urinary methylmalonic acid.[20] The mechanism of cobalamin responsiveness may be explained by distinct mutations in the $M M U T$ gene, same as the paper of tetrahydrobiopterin responsive phenylalanine hydroxylase deficiency.[21] In our patient, two mutations, c. $1292 \mathrm{~T}>\mathrm{C}$ and c.1808G $>\mathrm{A}$, were identified in a compound heterozygous state, in a potentially recessive pattern of inheritance. The novel mutation, c.1292T $>C$ (p.Met431Thr) is located in the linker region (residues 423-577).[22] This region is interconnects the $\mathrm{N}$-terminal domain and the C-terminal domain, which are the binding sites for substrate methylmalonyl$\mathrm{CoA}$ and cofactor adenosylcobalamin, respectively. The linker region in itself does not contribute residues to either the ligand binding pockets or the catalytic center. In this less functional region, the nearby mutations, c.1276G $>\mathrm{A}$ (p.Gly426Arg)[22] and c.1277G >A (p.Gly426Glu)[19] were reported as mut- MMA, which is the milder phenotype with some residual activity in the presence of high concentrations of adenosylcobalamin. The second variant c.1808G $>\mathrm{A}$ was reported as $m u t^{0} \mathrm{MMA},[17-$ 19] although predicted as non-detrimental by all the in silico algorithms. This mutation lay on the last nucleotide of exon 10, and conferred abnormal splicing detected on the cDNA level (c.1747_1808del, p.Val583GlyFs ${ }^{\star}$ ). These data suggest that the former mutation, c.1292T>C (p.Met431Thr) is responsible for the cobalamin responsiveness described in this case. Therefore, on the basis of the above findings, our patient's disease was diagnosed as mut MMA.

Although screening using MS/MS reveals suspected patients with MMA by elevated C3,[9-10] the diagnostic process after a primary screening is typically complex.[2] To distinguish between MMA and propionic acidemia, elevated levels of methylmalonic acid, 3-hydroxypropionic acid, and methylcitric acid should be detected by urinary organic acid analysis. Isolated elevation of urinary methylmalonic acid without hyperhomocysteinemia and low cobalamin level is caused by MMA, including deficiency of MCM, Cbl A, Cbl B, and Cbl D. Cobalamin responsiveness should also be assessed in every MMA patient by repeating a urinary organic acid analysis or acylcarnitine profile analysis. Traditionally, this process takes time and often causes treatment delay. In addition, NBS using MS/MS creates a high number of false-positive cases, leading to frequent recalls, family anxiety, long time to diagnosis, and unnecessary medical cost. $[12,23]$ Decreased gestational age and increased body weight are associated with increased C3, which enhances the false-positive rate.[24]

Recently, to simplify the second-tier testing, and to reduce the false-positive rate in NBS, effective methods have been reported. For example, LC/MS/MS revealed the simultaneous determination of tHcy, methionine, methylmalonic acid, and methylcitric acid in plasma or serum,[13] and another paper reported that LC/MS/MS detected methylmalonic acid, 3-hydroxypropionic acid, and methylcitric acid in the DBS as in primary screening.[12] DNA analysis using next-generation sequencing is also beneficial.[11] Although these second-tier tests decrease time to diagnosis, and the emotional and financial burdens of unneeded medical precautions, they are currently only used as assays in NBS, not as examinations for evaluating treatment. Wang et al reported good correlation between DBS methylmalonic acid concentration by LC/MS/MS and urinary methylmalonic acid concentration by gas chromatography mass spectrometry. In addition, they mentioned the promise of LC/MS/MS for the follow-up of children with MMA.[25] However, they did not use LC/MS/MS in clinical practice for patients with MMA.

Patients with MMA may suffer from metabolic strokes and hyperammonemia during the course of treatment, even if adequate at the time of diagnosis. Movement disorders, spastic quadraparesis, or paraparesis are potential sequelae of metabolic strokes.[4] A history of hyperammonemia was related to lower measures for full-scale IQ.[7] Therefore, before triggering a metabolic attack, it is important to evaluate whether the current management method is valid.

The LC/MS/MS method, particularly using labeled molecules as internal standards, is characterized by increased accuracy 
and precision. Persichilli et al showed that several LC/MS/MS methods have been reported for tHcy determination showing superior analytical quality and high throughput, some of which are also applicable to large routines.[26]

Herein, we described a simple and accurate LC/MS/MS method with a separation column, which can provide the acylcarnitine profile, methylmalonic acid level, and tHcy level in the DBSs in the same sample. This method revealed cobalamin responsiveness of the patient with MMA earlier than would have been possible using other methods. Based on the current data, this method seems to be applicable for the follow-up of the treatment of MMA patients. Furthermore, this rapid determination in DBS allows for better second-tier tests that would allow screening of combined MMA/homocystinuria conditions (cbl C, D, F, J), and evaluates cobalamin responsiveness in the follow-up of positive newborn screen cases for MMA. However, this should be confirmed with more experience with a larger number of cases and a wider spectrum of disorders.

\section{Conclusion}

This article described a simple, rapid, and accurate LC/MS/MS method, which can be applied not only to screen for MMA, but also to develop therapeutic strategy.

\section{Acknowledgements}

We thank Dr. Yasuhiro Maeda (Center for Research Promotion and Support, Fujita Health University) for analysis of MCM activity.

We also thank Dr. Osamu Sakamoto (Department of Pediatrics, Tohoku University School of Medicine) and Dr. Hideo Sasai and Dr. Toshiyuki Fukao (Department of Pediatrics, Graduate School of Medicine, Gifu University) for interpretation of and comments about mutation analysis.

This research was partially supported by the Practical Research Project for Rare/Intractable Diseases from Japan Agency for Medical Research and Development, AMED (Grant Number JP17ek0109276).

\section{Funding}

This research received no other grants from any funding agency in the public, commercial, or not-for-profit sectors.

\section{Declaration of Conflicting Interests}

The authors declared no potential conflicts of interest with respect to the research, authorship, and/or publication of this article.

\section{Supplementary Material}

The following online material is available for this article:

Table S1 - LC conditions for analysis of tHcy

Table S2 - LC conditions for analysis of methylmalonic acid

\section{References}

1. Baumgartner MR, Hörster F, Dionisi-Vici C, et al. Proposed guidelines for the diagnosis and management of methylmalonic and propionic acidemia. Orphanet J Rare Dis. 2014;9:130. doi:10.1186/S13023-014-0130-8

2. Keyfi F, Talebi S, Varasteh AR. Methylmalonic Acidemia Diagnosis by Laboratory Methods. Rep Biochem Mol Biol. 2016;5(1):1-14.

3. Hörster F, Baumgartner MR, Viardot C, et al. Long-term outcome in methylmalonic acidurias is influenced by the underlying defect (mut0, mut-, cblA, cblB). Pediatr Res. 2007;62(2):225-230. doi:10.1203/pdr.0b013e3180a0325f

4. Fraser JL, Venditti CP. Methylmalonic and propionic acidemias: clinical management update. Curr Opin Pediatr. 2016;28(6):682-693. doi:10.1097/mop.0000000000000422

5. Prada CE, Al Jasmi F, Kirk EP, et al. Cardiac disease in methylmalonic acidemia. J Pediatr. 2011;159(5):862-864. doi:10.1016/j.jpeds.2011.06.005.

6. Spada M, Calvo PL, Brunati A, et al. Early Liver Transplantation for Neonatal-Onset Methylmalonic Acidemia. Pediatrics. 2015;136(1):e252-256. doi:10.1542/ peds.2015-0175

7. O'Shea CJ, Sloan JL, Wiggs EA, et al. Neurocognitive phenotype of isolated methylmalonic acidemia. Pediatrics. 2012;129(6):e1541-1551. doi:10.1542/peds.2011-1715

8. MacFarland S, Hartung H. Pancytopenia in a patient with methylmalonic acidemia. Blood. 2015;125(11):1840. doi:10.1182/blood-2014-11-609479

9. Heringer J, Valayannopoulos V, Lund AM, et al. Impact of age at onset and newborn screening on outcome in organic acidurias. J Inherit Metab Dis. 2016;39(3):341-353. doi:10.1007/S10545-015-9907-8

10. Chace DH, Hannon WH. Impact of second-tier testing on the effectiveness of newborn screening. Clin Chem. 2010;56(11):1653-1655. doi:10.1373/clinchem.2010.153494

11. Peng G, Shen P, Gandotra N, et al. Combining newborn metabolic and DNA analysis for second-tier testing of methylmalonic acidemia. Genet Med. 2019;21:896-903. doi:10.1038/s41436-018-0272-5

12. Monostori P, Klinke G, Richter S, et al. Simultaneous determination of 3-hydroxypropionic acid, methylmalonic acid and methylcitric acid in dried blood spots: Secondtier LC-MS/MS assay for newborn screening of propionic acidemia, methylmalonic acidemias and combined remethylation disorders. PLoS One. 2017;12(9):e0184897. doi:10.1371/journal.pone.0184897 
13. Fu X, Xu YK, Chan P, Pattengale PK. Simple, Fast, and Simultaneous Detection of Plasma Total Homocysteine, Methylmalonic Acid, Methionine, and 2-Methylcitric Acid Using Liquid Chromatography and Mass Spectrometry (LC/MS/MS). JIMD Rep. 2013;10:69-78. doi:10.1007/8904_2012_205

14. Turgeon C, Magera M, Cuthbert C, et al. Determination of Total Homocysteine, Methylmalonic Acid, and 2-Methylcitric Acid in Dried Blood Spots by Tandem Mass Spectrometry. Clin Chem. 2010;56(11):1686-1695. doi:10.1373/clinchem.2010.148957

15. Gotoh K, Nakajima Y, Tajima G, et al. Assay for methylmalonyl coenzyme A mutase activity based on determination of succinyl coenzyme A by ultrahighperformance liquid chromatography tandem mass spectrometry. Anal Bioanal Chem. 2015;407(18):52815286. doi:10.1007/s00216-015-8753-8

16. Matsumoto I, Kuhara T. A new chemical diagnostic method for inborn errors of metabolism by mass spectrometryrapid, practical, and simultaneous urinary metabolites analysis. Mass Spectrom Rev. 1996;15(1):43-57. doi:10.1002/ (sici)1098-2787(1996)15:1<43::aid-mas3>3.0.co;2-b

17. Martínez MA, Rincón A, Desviat LR, Merinero B, Ugarte M, Pérez B. Genetic analysis of three genes causing isolated methylmalonic acidemia: identification of 21 novel allelic variants. Mol Genet Metab. 2005;84(4):317-325. doi:10.1016/j.ymgme.2004.11.011

18. Merinero B, Pérez B, Pérez-Cerdá C, et al. Methylmalonic acidaemia: examination of genotype and biochemical data in 32 patients belonging to mut, cblA or cblB complementation group. J Inherit Metab Dis. 2008;31(1):55-66. doi:10.1007/ s10545-007-0667-y

19. Forny P, Schnellmann AS, Buerer C, et al. Molecular Genetic Characterization of 151 Mut-Type Methylmalonic Aciduria
Patients and Identification of 41 Novel Mutations in MUT. Hum Mutat. 2016;37(8):745-754. doi:10.1002/humu.23013

20. Scalais E, Osterheld E, Geron C, et al. Parenteral hydroxocobalamin dose intensification in five patients with different types of early onset intracellular cobalamin defects: Clinical and biochemical responses. JIMD Rep. 2019;49(1):70-79. doi:10.1002/jmd2.12055

21. Kure S, Hou DC, Ohura T, et al. Tetrahydrobiopterinresponsive phenylalanine hydroxylase deficiency. J Pediatr. 1999;135(3):375-378. doi:10.1016/s0022-3476(99)70138-1

22. Worgan LC, Niles K, Tirone JC, et al. Spectrum of mutations in mut methylmalonic acidemia and identification of a common Hispanic mutation and haplotype. Hum Mutat. 2006;27(1):31-43. doi:10.1002/humu.20258

23. Matern D, Tortorelli S, Oglesbee D, Gavrilov D, Rinaldo P. Reduction of the false-positive rate in newborn screening by implementation of MS/MS-based second-tier tests: the Mayo Clinic experience (2004-2007). J Inherit Metab Dis. 2007;30(4):585-92. doi:10.1007/s10545-007-0691-y

24. Peng G, de Fontnouvelle CA, Enns GM, Cowan TM, Zhao $\mathrm{H}$, Scharfe C. Elevated methylmalonic acidemia (MMA) screening markers in Hispanic and preterm newborns. Mol Genet Metab. 2019;126(1):39-42. doi: 10.1016/j. ymgme.2018.11.006

25. Wang Y, Sun Y, Jiang T2. Clinical Application of LC-MS/ MS in the Follow-Up for Treatment of Children with Methylmalonic Aciduria. Adv Ther. 2019;36(6):1304-1313. doi:10.1007/s12325-019-00955-0

26. Persichilli S, Gervasoni J, Iavarone F, Zuppi C, Zappacosta B. A simplified method for the determination of total homocysteine in plasma by electrospray tandem mass spectrometry. J. Sep. Sci. 2010;33(20):3119-3124. doi:10.1002/jssc.201000399 